\title{
Clinical and laboratory investigation on the recurrence of the umbilical hernia after herniorrhaphy in bovine calves
}

\author{
Md. Mehedi Jaman ${ }^{1}$, Pravin Mishra ${ }^{1}$, Marzia Rahman ${ }^{2}$ and ${ }^{\otimes}$ Md. Mahmudul Alam ${ }^{1}$ \\ ${ }^{1}$ Department of Surgery and Obstetrics, Bangladesh Agricultural University, Mymensingh-2202, Bangladesh \\ ${ }^{2}$ Department of Microbiology and Hygiene, Bangladesh Agricultural University, Mymensingh-2202, Bangladesh
}

\begin{tabular}{l}
\hline ARTICLE INFO OPENOACcES \\
\hline Article history: \\
Received: 16 July 2018 \\
Accepted: 05 December 2018 \\
Published: 31 December 2018 \\
\hline Keywords: \\
Umbilical hernia; Herniorrhaphy; \\
Recurrence; Infection \\
\hline Correspondence: \\
Md. Mahmudul Alam \\
凶: mahmuddso2004@gmail.com
\end{tabular}

\begin{abstract}
This study was performed to investigate the recurrence of the umbilical hernia in bovine calves at Veterinary Teaching Hospital (VTH), Bangladesh Agricultural University, Mymensingh. A total of 34 animals were admitted to VTH diagnosed to have the umbilical hernia, among them 13 were recurrent cases. The diagnosis of umbilical hernia was based on the disease history, clinical signs, palpation, needle exploration, and auscultation. The risk factors like age, sex, breed, body weight, the season of affection, size of the hernial ring, suture materials, error in suture patterns and involvement of infections in the recurrence were considered. Infected samples were collected from recurrent patients with infected umbilicus region, and subjected to bacteriological analysis. We found the recurrence percentage was higher in the crossbred (44\%) than that in the indigenous $(22.22 \%)$ calves. It was also observed that recurrence percentage was higher in male $(42.11 \%)$ than those in female $(33.33 \%)$ patients. The recurrence percentage was also higher in calves aged below three months $(40.74 \%)$ than the calves of above three months $(28.57 \%)$. It was found that recurrence percentage was most frequent in summer $(46.15 \%)$ and the least in the winter $(30 \%)$. Among 13 recurrent bovine calves after herniorrhaphy, three calves returned to VTH with umbilical abscess, six calves recurred due to non-infectious causes like failure of suture materials, errors in suture pattern or management fault and 4 calves recurred with both umbilical abscess and non-infectious causes. From this study, it may be concluded that synthetic absorbable suture materials with secured suture pattern and good postoperative management may help in reducing the recurrence of an umbilical hernia.
\end{abstract}

\section{Introduction}

Congenital disorders in calves have been increasing alarmingly with the increase of crossbred animals. Umbilical hernia is one of the major congenital disorders causing mortality in calves (Nath et al., 2016). Herniorrhaphy is the best acceptable treatment of this congenital anomaly. Recently, recurrence of umbilical hernia after herniorrhaphy has appeared as a common event (Rahman et al., 2017). The recurrence of umbilical hernia causes irreparable loss to the farmers. Further, operational and medicinal cost is more than first time. Moreover, recur patient is ineligible for calving and draught purpose on the eye of farmers. So, the recurrent calf is either culled or sold.

Recurrent calf may be infected or non-infected (Roger and David, 2011). In case of infection, there is presence of pus on the affected site. Infection of the umbilicus or umbilical cord remnants often occurs in the neonatal period as a result of environmental contamination, but the umbilicus may also be seeded with bacteria from a generalized septicemia/bacteremia (Fubini, 2016). In case of non-infected calf, suture materials may play a vital role for recurrence because of premature absorption of the thread leading to the disruption of hernia ring.
A lot of research works regarding correction of umbilical hernia have been documented (Rahman et al., 2017; Salim et al., 2015; Sutradhar et al., 2009) but the researches on the recurrence of umbilical hernia are rarely found in Bangladesh. Considering the above facts, the present study was undertaken to investigate the incidence of the recurrence of umbilical hernia and the etiological factors involved in the recurrence of umbilical hernia in bovine calves after surgical intervention. Besides, finding out the pathogenic organisms associated with the infection and the choice of appropriate suture materials and suture patterns for the prevention of the recurrence of umbilical hernia are also our research goals.

\section{Materials and Methods}

The animals with umbilical hernia brought to the Veterinary Teaching Hospital (VTH), Bangladesh Agricultural University (BAU) were considered as the experimental animal.

This study was undertaken during the period from December 2016 to November 2017.

Once the patient arrived at VTH, BAU, the parameters like age, sex, breed, bodyweight, season (time) of 
affection, size of hernia ring and new or recurrent patient were recorded carefully.

\section{Treatment of the new patient}

In new patient, herniorrhaphy was performed with the following procedure:

After 24 hours of fasting the animal was controlled in dorsal recumbence and the operative area was prepared with routine aseptic procedure. Diazepam (Sedil ${ }^{\circledR} 2 \%$; Square Pharmaceuticals, Bangladesh) at a dose rate of $0.4 \mathrm{mg} / \mathrm{kg}$ was administered intramuscularly to sedate the patient. Later, infiltration anaesthesia was followed using $2 \%$ lignocaine hydrochloride (Jasocaine ${ }^{\circledR}$, Jayson Pharmaceuticals Ltd., Dhaka, Bangladesh) in an inverted ' $\mathrm{V}$ ' shaped manner from cranial to caudal aspect of hernial ring. An elliptical incision was made through the skin and each side of the swelling. After blunt dissection, incision was made on the sac to check for internal adhesion if any. Then the sac was incised just above the ring and contents were pushed back to the abdomen. The ring was scratched and closed by placing a series of overlapping mattress sutures through its edges. Muscles were sutured layer by layer using chromic catgut with simple continuous suture pattern. The skin flaps were apposed by simple interrupted mattress sutures using non-absorbable material.

\section{Approach to recurrent patient}

In case of recurrent animal, history was taken regarding treatment protocol, management of the patient, suture material and suture pattern by interviewing the owner or from the register book at VTH, BAU. The site was inspected carefully whether there was any infection like abscess formation, oozing of pus from umbilicus etc. In our study, we found both infected and non-infected recurrent calves. In case of non-infected calves, the patient was only corrected through further surgical operation by myomattress suture pattern with chromic catgut or Vicryl ${ }^{\circledR}$. Following surgery, advices were given to the owner for careful management. But in case of infected patient, pus was collected from the site of infection to identify pathogen involved in the infection. After a course of systemic antibiotic, hernia was repaired using routine procedure as stated above.

\section{Culture of the sample}

\section{a) Spread plate method}

In these steps, samples were subjected to 10 -fold serial dilutions and a small aliquot was poured to an agar plate. The samples were then distributed evenly over the surface of Mannitol-salt agar media, MacConkey agar media and Blood agar media with a bent glass rod. Glass rod was sterilized by dipping into $70 \%$ alcohol solution and then passed it quickly through the Bunsen burner flame. When all alcohol was burned off and rod was air cooled, the plate was streaked up and down several times. Then the plates were inoculated at $37{ }^{\circ} \mathrm{C}$ for 24 hours (overnight). After incubation, the plate was examined for the growth of bacteria as colonies on the surface of culture medium (Fig. 1. A, B, C, D, E).
A

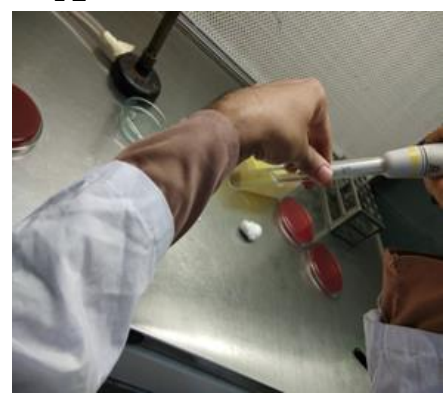

D

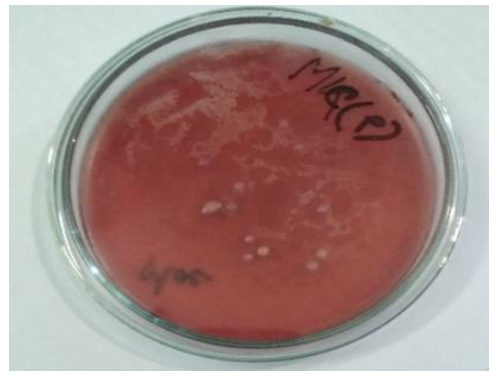

Pseudomonas spp.
B

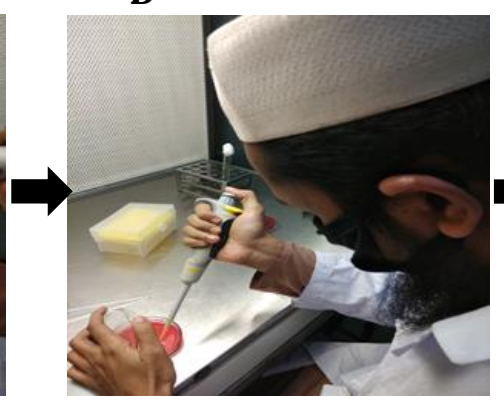

E
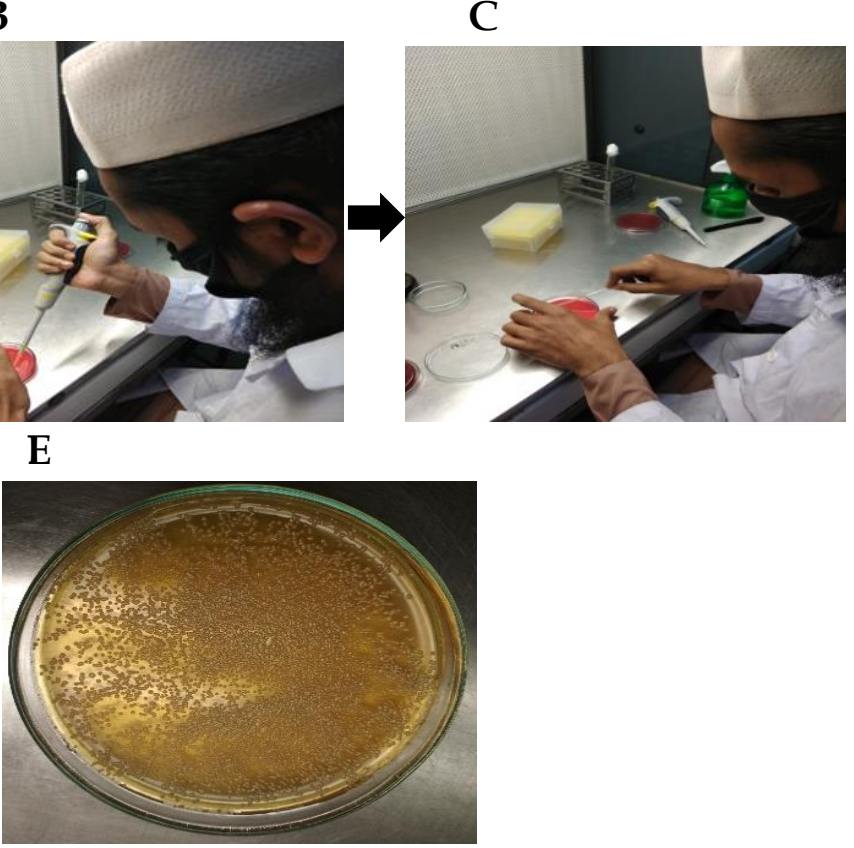

Staphylococcus aureus

Fig. 1. Culture of the samples according to spread plate method (A, B, C) and Colony of Pseudomonas and Staphylococcus (D, E) in the Mannitol-salt agar and MacConkey agar media respectively 
b) Streak plate method

It was the method of culturing aerobic bacteria by streaking the surface of a solid medium in a petri dish with an inoculating loop. This inoculating loop was sterilized by heat then cooled. Sample was taken with a sterilized loop. The sample was placed at one point on solid surface of Mannitol-salt agar media, MacConkey agar media and Blood agar media. The samples were streaked several times on the surface. The culture media were inoculated at $37^{\circ} \mathrm{C}$ for 24 hours (overnight) in anaerobic condition. After incubation, the plate was examined for the growth of bacteria as colonies on the surface of culture medium (Fig. 2. A, B, C, D, E).

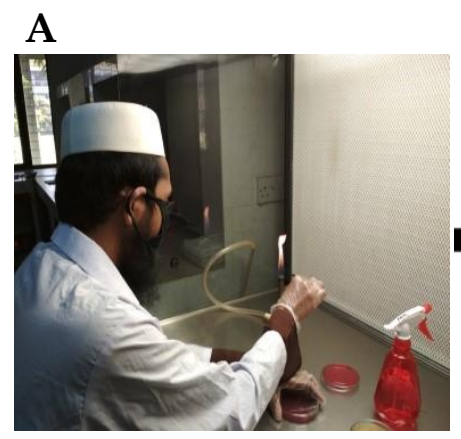

D

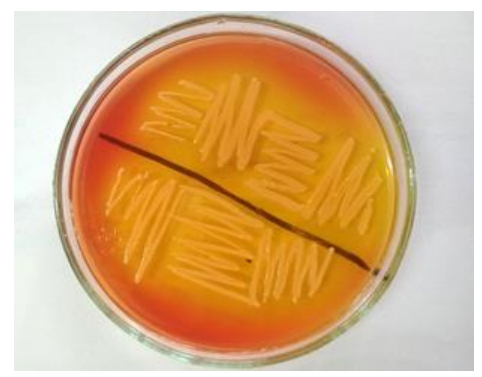

Staphylococcus aureus
B

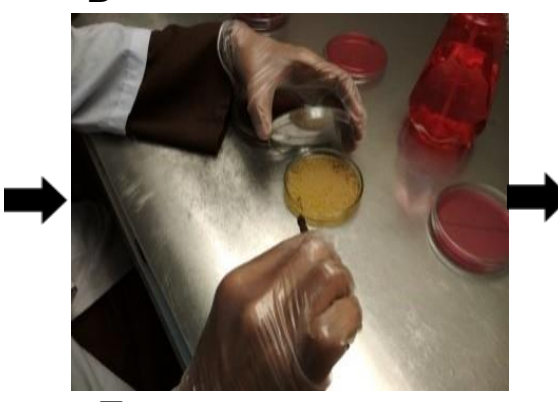

E

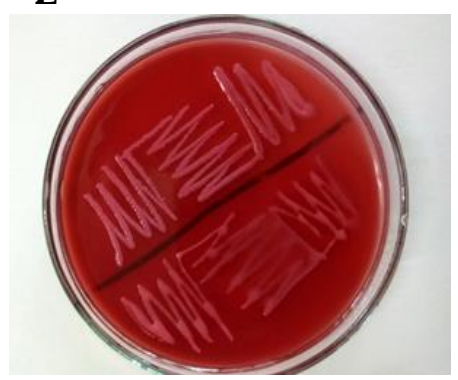

Pseudomonas spp.

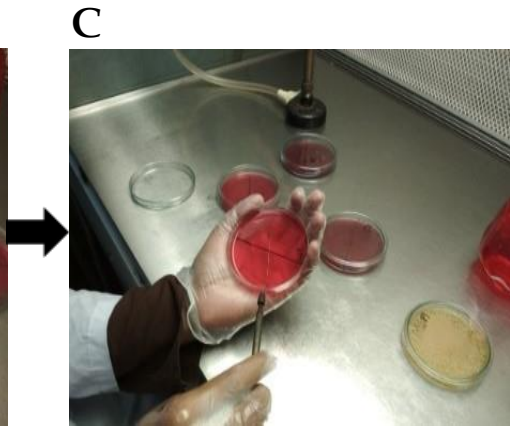

C

.

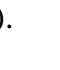


open, abdominal contents can protrude through the defect resulting in an umbilical hernia (Dennis et al., 1997). Many umbilical hernias are secondary to umbilical sepsis. This may occur as isolated defects or may be associated with defects of other parts of the body (Steenhold et al., 2004). In the study area, the overall recurrence of umbilical hernia was as high as $38.24 \%$ (13 calves out of 34) after herniorrhaphy.

\section{Recurrence based on breed}

The hernia has been reported to be hereditary in origin (Herrmann, 2001). In this study, we found higher recurrence rate in the crossbred than that in the indigenous breed following surgery. The breed predisposition on the recurrence of umbilical hernia in calves is shown in Table 1. Among the 34 affected calves, 2 out of 9 affected indigenous calves were recurrent and 11 patients were returned to the hospital with reformation of umbilical hernia out of 25 crossbred calves. Breed difference in the recurrence of umbilical hernia was evident. The recurrence percentage was $22.22 \%$ and $44 \%$ in indigenous and crossbred calves, respectively.

Table 1. Recurrence of umbilical hernia following surgery based on breed in bovine calves

\begin{tabular}{lccc}
\hline Breed & $\begin{array}{c}\text { Hernia } \\
\text { operated } \\
\text { patients }\end{array}$ & $\begin{array}{c}\text { Recurrent } \\
\text { animals }\end{array}$ & $\begin{array}{c}\text { Recurrence } \\
\text { percentage } \\
(\%)\end{array}$ \\
\hline Indigenous breed & 9 & 2 & 22.22 \\
Crossbred & 25 & 11 & 44 \\
Total & 34 & 13 & 38.24 \\
\hline
\end{tabular}

This finding is similar to the occurrence of umbilical hernia observed by Rings (1995), Steenhood and Hernandez (2004), who stated that umbilical hernias were the most common birth defects in calves especially in Holstein Frisian breeds indicating genetic in nature.

\section{Recurrence based on sex}

In our study, the recurrent rate was higher in male than in female calves (Table 2). Among 34 surgically treated calves, 9 male calves out of 19 and 3 female calves out of 15 were recurrent; the recurrence percentage in male and female were $42.11 \%$ and $33.33 \%$ respectively.

Table 2. Recurrence of umbilical hernia based on sex in bovine calves

\begin{tabular}{cccc}
\hline Sex & $\begin{array}{c}\text { Surgically treated } \\
\text { hernial patients }\end{array}$ & $\begin{array}{c}\text { Recurrent } \\
\text { animals }\end{array}$ & $\begin{array}{c}\text { Recurrence } \\
\text { percentage } \\
(\%)\end{array}$ \\
\hline Male & 19 & 8 & 42.11 \\
Female & 15 & 5 & 33.33 \\
Total & 34 & 13 & 38.24 \\
\hline
\end{tabular}

This finding disagrees with the finding of Mazakazu (2005), who observed that gender had effect on occurrence of umbilical hernia and female showed a higher incidence than male. In our study, higher recurrence in male may be due to the contamination of operated site with urine during urination or become wet due to improper management. But, female may be free from this sort of danger due to anatomical location.

\section{Recurrence based on age}

Generally, umbilical hernia is mostly occurred between 1 and 3 months of age while calves below 1 month and those above 3 months are less commonly affected (Gadre et al., 1989). In our study, the recurrence rate was higher in the calves aged three months and bellow three months than calves aged above three months (Table 3). Among the 34 surgically corrected hernial calves, 2 out of 7 calves $(28.57 \%)$ aged above three months were recurrent and 11 out of 28 calves $(40.74 \%)$ were between 1 to 3 months of age.

Table 3. Recurrence of umbilical hernia based on age in bovine calves

\begin{tabular}{lccc}
\hline $\begin{array}{l}\text { Age of the } \\
\text { animal }\end{array}$ & $\begin{array}{c}\text { Surgically corrected } \\
\text { umbilical hernial } \\
\text { patients }\end{array}$ & $\begin{array}{c}\text { Recurrent } \\
\text { animals }\end{array}$ & $\begin{array}{c}\text { Recurrence } \\
\text { percentage } \\
(\%)\end{array}$ \\
\hline$\leq 3$ months & 27 & 11 & 40.74 \\
$>3-6$ months & 7 & 2 & 28.57 \\
Total & 34 & 13 & 38.24 \\
\hline
\end{tabular}

In this connection, Jettennavar et al., (2010) conducted a study in 18 commercial dairy farms in New York, 15\% of heifer calves had umbilical hernias during the first 3 months of age.

\section{Recurrence based on season}

We studied if there was any effect of season on the recurrence of umbilical hernia and found that the recurrence was higher in the summer $(46.15 \%)$ than in the rainy $(36.36 \%)$ and winter $(30 \%)$ seasons (Table 4$)$.

Table 4. Recurrence of umbilical hernia based on season in bovine calves

\begin{tabular}{lccc}
\hline Season & $\begin{array}{c}\text { Surgically corrected } \\
\text { umbilical hernial } \\
\text { patients }\end{array}$ & $\begin{array}{c}\text { Recurrent } \\
\text { animals }\end{array}$ & $\begin{array}{c}\text { Recurrence } \\
\text { percentage } \\
(\%)\end{array}$ \\
\hline $\begin{array}{l}\text { Winter (October- } \\
\text { January) } \\
\begin{array}{l}\text { Summer } \\
\text { (February-May) }\end{array}\end{array}$ & 10 & 3 & 30 \\
$\begin{array}{l}\text { Rainy (June- } \\
\text { September) }\end{array}$ & 11 & 6 & 46.15 \\
\hline
\end{tabular}

The recurrence of umbilical hernia has been found to occur throughout the year (Nath, 2016). In our study, recurrence of umbilical hernia was higher $(46.15 \%)$ in the summer (February-May) whereas the lower incidence $(30 \%)$ was recorded in the winter season (June-September). This observation was agreeable with earlier reports of occurrence of umbilical hernia (Dehoux, 1992; Samad et al., 2002). This may have happened because the occurrence of surgical site infection (SSI) is greater in summer season than other seasons. According to Durkin et al. (2016), the rates of SSI remained higher during the summer after stratification by pathogen. Specifically, SSI due to either 
Gram-positive cocci like Staphylococcus aureus or Gram-negative bacilli which were more common during the summer season.

\section{Recurrence based on suture and suture patterns}

Suture pattern plays an important role in the success of hernia repair. The recurrence of umbilical hernia based on suture and suture patterns in bovine calves is shown in Table 5.

Table 5. Recurrence of umbilical hernia based on suture and suture patterns in bovine calves

\begin{tabular}{lccc}
\hline Suture patterns & $\begin{array}{c}\text { Surgically corrected } \\
\text { umbilical hernial } \\
\text { patients }\end{array}$ & $\begin{array}{c}\text { Recurrent } \\
\text { animals }\end{array}$ & $\begin{array}{c}\text { Recurrence } \\
\text { percentage } \\
(\%)\end{array}$ \\
\hline $\begin{array}{l}\text { Myomattress suture } \\
\text { pattern with chromic }\end{array}$ & 18 & 4 & 22.22 \\
$\begin{array}{l}\text { catgut } \\
\text { Unknown suture }\end{array}$ & 16 & 9 & 56.25 \\
$\begin{array}{l}\text { pattern with chromic } \\
\text { catgut }\end{array}$ & & & \\
Total & 34 & 13 & 38.24 \\
\hline
\end{tabular}

Recurrence percentage was $38.24 \%$ in both suture repair groups whereas it was higher in animal treated with unknown suture pattern with chromic catgut $(56.25 \%)$ than animal treated with myomattress suture pattern with chromic catgut $(22.22 \%)$. Very similar results have been reported by Venclauskas (2008), that recurrence rate was up to $19-54 \%$ with horizontal suture pattern used to repair the ring. In our study, we found that failure of umbilical suture as chromic catgut was more frequent. According to Mosbah, and Karrouf (2006), the recurrent recorded cases may be due to the decrease in the tensile strength of the synthetic absorbable suture material (chromic catgut) before complete healing of the hernial ring. Ritchie (1990) said that chromic catgut should be avoided in avian surgery because of developed a marked granulocytic inflammatory response due to catgut that diminished the strength of the suture during the period of healing and possible to recur.

We used Vicryl ${ }^{\circledR}$ in one case and obtained good result that there was no recurrence (data not shown). It may be due to excellent properties of Vicryl $^{\circledR}$ as it is nonantigenic, does not swell when wet, causes minimal tissue reaction, ensures good handling and knot security (Hendrickson, 2013). In case of suture patterns, recurrence was lower in myomattress suture pattern than others. This observation is agreed with Ghalot et al (1995), who stated that about $8.33 \%$ umbilical hernia were recurrent after treatment with myomattress pattern using catgut, whereas $50 \%$ recurrence were found in horizontal mattress pattern. Relatively, higher incidence of recurrence in horizontal mattress pattern is due to direct pressure of bowel on suture line during healing process (Ghalot et al, 1995).
Relationship between hernial ring diameter and weight of bovine calves

In the study, it was found that the hernial ring's size of recurrent animals were 2 to $4.5 \mathrm{~cm}$. A relationship exists between diameter of hernial ring's size and bodyweight of calves and the hernial diameter increases with the bodyweight of calves. In the Pearson's productmoment correlation test with $5 \%$ level of confidence, the p-value $=0.0001356$. So, accroding to hypothesis, we can say that the relation between hernial ring diameters and weight of bovine calves is significant. The relationship between hernial ring diameters and weight of bovine calves is shown in Fig. 4.

In the current study, we found that the recurrent hernial ring diameters were between 2 to $4 \mathrm{~cm}$ and there was a relationship between hernial ring diameter and weight of bovine calves that the hernial diameter increases according to the weight of calves. The study is similar to the hernia recurrence in human according to Venclauskas (2008), that recurrence rate was higher in patients whose hernia size was $>2 \mathrm{~cm}$ and patient's bodyweight was $>30 \mathrm{~kg}$ comparing with hernia size of $<2 \mathrm{~cm}$ and $<30 \mathrm{~kg}$ bodyweight.

\section{Bacterial invasion}

We found the recurrent calves with infected state as well. There was presence of pus in operation site. Among 13 recurrent calves, 3 calves were recurrent with hernial abscess. To identify bacterial involvment, samples were collected and cultured in microbiological laboratory in the Department of Microbiology and Hygiene, BAU. Pathogenic bacteria were identified based on cultural characteristics and Gram's staining reaction and these were Gram positive Staphylococcus aureus and and Gram negative rods like Pseudomonas spp. (Fig. 5).

Recurrent calf may be infected or non-infected. In our study, we found recurrence with infection $(23.08 \%)$ and recurrence without infection (46.15\%) and both infected and non-infected recurrence $(30.77 \%)$. We used suture materials such chromic catgut (32 calves), prosthetic mesh (one calf, data not shown), only Vicryl ${ }^{\circledR}$ (one calf) and found that patient treated with Vicryl ${ }^{\circledR}$ was free from infection. This finding is similar to Hendrickson (2013), who stated that Vicryl ${ }^{\circledR}$ reduces bacterial attachment to the suture material and act as an antimicrobial impregnated suture. In case of infected recurrent calves, pathogenic agents were isolated from the lesion through microbiological culture namely Staphylococcus aureus and Pseudomonas spp. This observation was agreed with Fubini (2016) in case of umbilical infections, who stated that common bacteria isolate from umbilical infections in calves included Staphylococcus aureus, Pseudomonas spp. and Escherichia coli etc. 


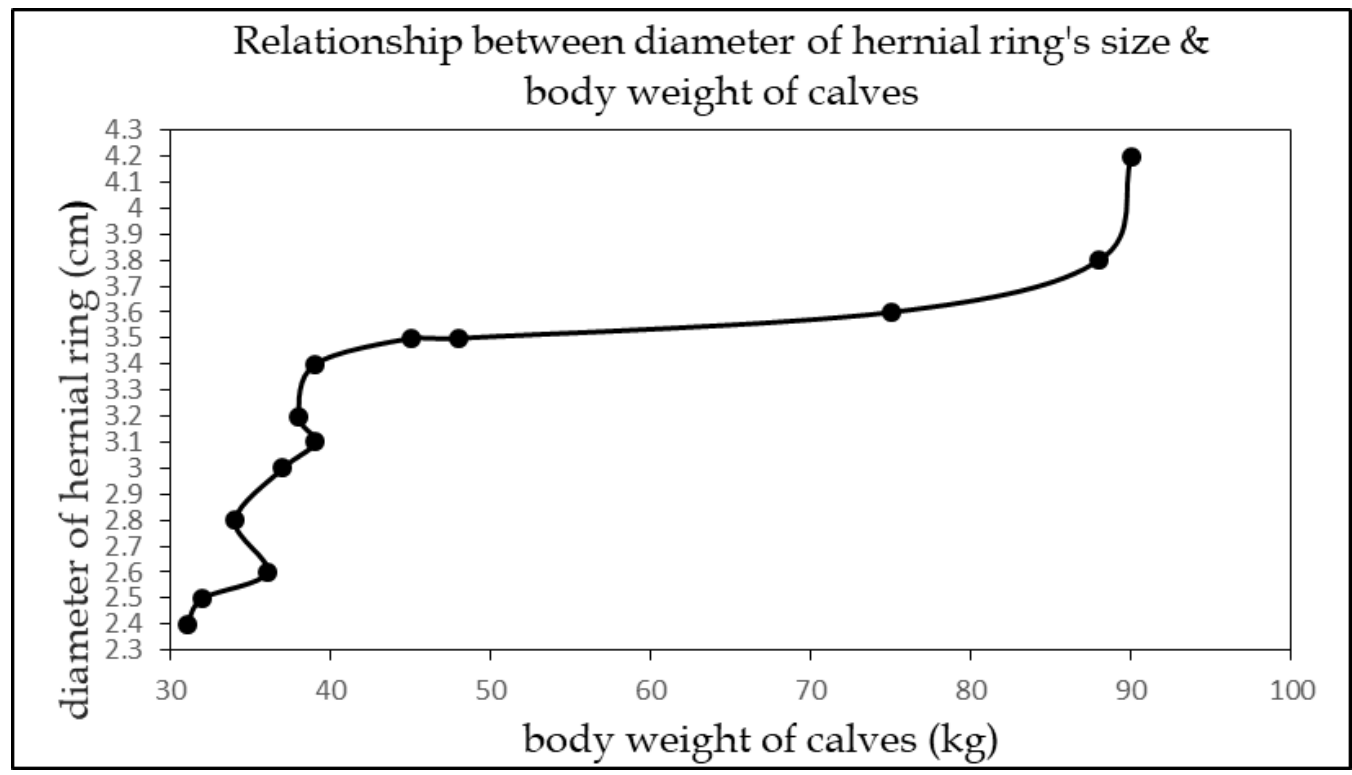

Fig. 4. Graphical representation on the relationship between diameters of hernial ring and body weight of bovine calves on the recurrence of umbilical hernia

A

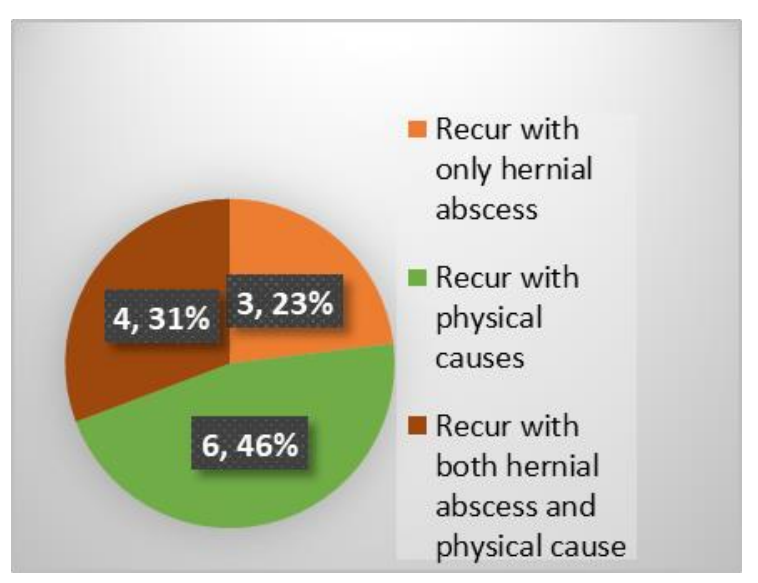

B

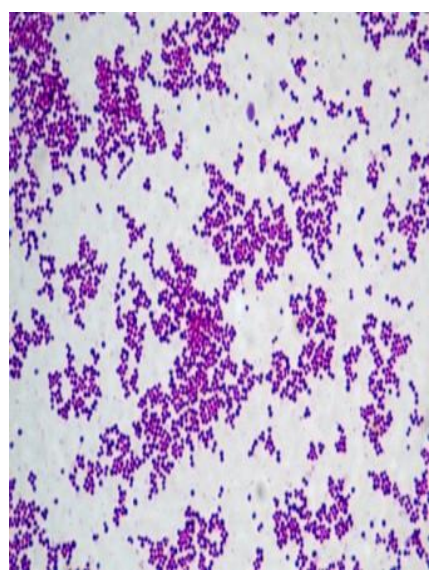

$\mathrm{C}$

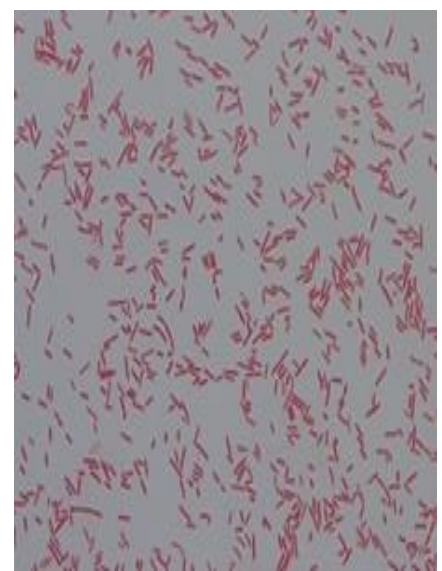

Fig. 5. Pathogenic involvement in the recurrence of umbilical hernia. (A) Percentage of recurrence associated with infection; (B, C) Result of Gram's staining reaction. (B) is showing Gram positive Staphylococcus aureus arranged in cluster and (C) is showing Gram negative small rod-shaped organisms arranged in single under light microscope (100X)

\section{Conclusion}

From investigation on recurrence of umbilical hernia of bovine calves after herniorrhaphy, the following conclusions can be made:

1) Anti-microbial impregnated and strong tensile strength suture (e.g. Vicryl ${ }^{\circledR}$ ) should be used to reduce both infection and recurrence of umbilical hernia.

2) Good suture pattern like overlapping myomattress suture should be chosen by surgeon to overcome error of suture pattern.

3) Awareness should be created among the farmers about calf management after herniorrhaphy and timely reference of recurrent cases of umbilical hernia to the respective veterinarian near the veterinary hospital for herniorrhaphy.

\section{Acknowledgements}

We thank National Science and Technology (NST) for providing financial support to conduct the research.

\section{References}

Cheesbrough, M. 2006. Gram's staining method. In: Cambridge University Press, Cambridge, UK (eds). District Laboratory Practice in Tropical Countries, 2nd Edn., ISBN-13: 9781139449298 PMCid:PMC2870630

Dehoux, J.P. 1992. Clinical observation of umbilical pathology in N'dama calves in Gabon. Bulletin of Animal Health and Production in Africa, 40: 209-211. 
Dennis, S.M. and Leipold, D.G 1997. Congenital hernia in calves. Journal of the American Veterinary Medical Association, 152: 999-1003.Durkin, M.J., Dicks, K.V., Baker, A.W., Lewis, S.S., Moehring, R.W., Chen, L.F., Sexton, D.J. and Anderson, D.J. 2015. Postoperative infection in spine surgery: does the month matter? Journal of Neurosurgery: Spine, 23(1): 128-134.

Fubini, S.L. and Norm, G.D. 2016. Miscellaneous abnormalities of the calf. In: Elsevier Inc., Philadelphia (eds). Farm Animal Surgery, 2nd Edn.19: 541-547.

Ghalot, T.K., Singh, J. and Sharma, C.K. 1995. Hernia of digital cushion in camel. Indian Veterinary Journal, 72: 887- 888.

Hendrickson, D.A. and Baird, A.N. 2013. Suture materials and needles. In: John Wiley \& Sons Inc.,Philadelphia(eds). Turner and Mcllwraith's Techniques in Large Animal Surgery, 4th Edn, pp. 61-70. Jettennavar, P.S., Kalmath, G.P. and Anilkumar, C.J. 2010. Ventral abdominal hernia in cattle. Veterinary World, 3(2): 93

Mazakazu, S. 2005. Umbilical hernia in Japanease black calves: A new technique and hereditary background. Journal of Live Medicine, 507: 543-547.

Mosbah, E. and Karrouf, G.I.A. 2006. Surgical management of certain umbilical affection in calves and foals. Minufiya Veterinary Journal, 26: 123-127.

Nath, S.K., Sen, A.B., Rahman, M.M., Das, S., Ersam, R. and Alam. M.R., 2016. Umbilical Hernia in Calves in Sylhet Region. International Journal of Advanced Multidisciplinary Research, 3(7): 19-25.

Rahman, M.M., Biswas, D. and Hossain, M.A. 2001. Occurrence of umbilical hernia and comparative efficacy of different suture materials and techniques for its correction in calves. Pakistan Journal of Biological Sciences, 4: 1026-1028.

Rahman, M.M., Sultana, S. and Ali, M.Z. 2017. Prevalence of umbilical hernia of calves and its risk factors at Tangail Sadar of Bangladesh. Asian-Australasian Journal of Bioscience and Biotechnology, 2 (2): 154-158.
Rings, D.M. 1995.Umbilical hernias, umbilical abscesses, urachal fistulas. Veterinary Clinics: Small Animal Practice, 11: 137-148. https://doi.org/10.1016/S0749-0720(15)30512-0

Ritchie, B.W., Doyle, J.E. and Harrison, G.J. 1990. Micro technique for the surgical management of avian diseases. Journal of Avian Medicine and Surgery, 32: 13-18.

Roger, B. and David, W. 2011.Conditions of umbilicus. In: Elsevier Inc., Philadelphia (eds). Color Atlas of Diseases and Disorders of Cattle, 3rd Edn., 2: 13 -16.

Salim, M., Hashim M.A., Jueyana, N.S., Arafat, Y.A., Dey R.K., Bag, M.A.S. and Islam, M.S. 2015. Prevalence of hernia evaluation of herniorrhaphy in calves. International Journal of Natural and Social Sciences, 2(4):35 - 43.

Samad, M.A., Islam, M.A. and Hossain, M.A. 2002.Patterns of occurrence of calf diseases in the district of Mymensingh in Bangladesh. Bangladesh Veterinary Journal, 36 (1-2): 01-05.

Steenholdt, C. and Hernandez, H. 2004. Risk factors for umbilical hernia in Holstein heifers during the first two months after birth. Journal of the American Veterinary Medical Association, 224: 431- 437 . https://doi.org/10.2460/javma.2004.224.1487

Sutradhar, B.C., Hossain, M.F., Das, B.C., Kim, G. and Hossain M.A. 2009. Comparison between open and closed methods of herniorrhaphy in calves affected with umbilical hernia. Journal of Veterinary Science,10(4): 343 - 347. https://doi.org/10.4142/jvs.2009.10.4.343 PMid:19934601 PMCid:PMC2807272

Venclauskas, L., Šilanskaite, J., Kanišauskaitė, J. and Kiudelis, M. 2008. Long-term results of incisional hernia treatment. Medicina (Kaunas), 43(11): $855-60$. https://doi.org/10.3390/medicina43110110 